\title{
EFECTO DEL ANÁLOGO DE BRASINOESTEROIDE (MH5) EN LA ACLIMATIZACIÓN DE LOS BROTES DE Vriesea PROPAGADOS EN SISTEMAS DE INMERSIÓN TEMPORAL
}

\author{
Iris Capote, Maritza Escalona, Marcos Daquinta, Danilo Pina, Justo González y Carlos Aragón \\ Laboratorio de Células y Cultivo Tejidos, Centro de Bioplantas, Universidad de Ciego de Ávila, \\ C. P: 69450. Cuba.
}

\begin{abstract}
RESUMEN
$\mathrm{L}$ as bromelias son plantas ornamentales muy atractivas por la coloración de sus hojas y la belleza de sus inflorescencias. Dentro de ellas las Vrieseas es uno de los géneros de mayor interés comercial. Cuando se desean introducir nuevos híbridos en el mercado, las técnicas de propagación tradicional son insuficientes, por lo que resultan de gran interés las técnicas de propagación in vitro. Con el objetivo de establecer un protocolo para la aclimatización de brotes de Vriesea propagados in vitro en Sistemas de Inmersión Temporal se evaluó el efecto de la aplicación del brasinoesteroide MH5 en la supervivencia y calidad de los brotes. La integración de los resultados constituyen los procedimientos elementales para un protocolo de aclimatización de brotes de Vriesea propagados con el empleo de la técnica de inmersión temporal.
\end{abstract}

Palabras claves: bromelias, reguladores del crecimiento, ornamentales.
Abstract

$\mathrm{B}$ romeliads are very atractive ornamental plants because of their leaves coloration and the beautiful of its inflorescense. Among these plants, the Vrieseas is a genere of great commercial interest. When we wish to introduce new hybrids in the trade market, the technique of tradicional propagation are limited, for its the technique of in vitro propagation have great interest. With the aim of stablishing an in vitro aclimatization protocol of Vriesa shoots propagation in the Temporary Immersion System, the effect of brassinosteroids MH5 were evaluated for the survival and quality of shoots. The integration of these results are the elemental procedure for an in vitro aclimatization protocole of Vriesea shoots propagation with the use of temporary immersion technique.

Key words: bromeliads, growth regulators, ornamentals.

\section{INTRODUCCIÓN}

$\mathrm{L}$ as Bromelias se propagan de forma tanto natural a través de la vía sexual como de la asexual, pero ambas vías presentan problemas.

El desarrollo de las técnicas de micropropagación ha tenido resultados altamente ventajosos en la propagación rápida y con calidad de genotipos élites. Los protocolos de propagación in vitro de las Bromelias se han establecidos a partir del cultivo de ápices, yemas axilares y explantes de hojas provenientes de plantas adultas (Pierik y Sprenkles, 1991), del cultivo de plantas a partir de la germinación de las semillas in vitro (Mercier y Kerbauy, 1997; Alves y Guerra, 2001), y por la formación de yemas adventicias a partir de la parte basal de las hojas removidas de cultivos asépticos (Carneiro et al., 1999). Todos estos protocolos presentan como desventajas los bajos coeficientes de multiplicación, el alto costo de la mano de obra y la escasa posibilidad de automatización.

Recibido: Enero, 2009. Aceptado: Marzo, 2009. Publicado como ARTÍCULO en Ciencia y Tecnología 3: 29-33. 2009.
Los Brasinoesteroides (BRAS) son un grupo natural de esteroides polihidroxilados que fueron originalmente aislados del polen de Brassica napus (L.) en 1979 (Creelman y Mullet, 1997). Se caracterizan por producir la estimulación del crecimiento vegetal, el aumento de los rendimientos y la producción de biomasa en diferentes cultivos y el aceleramiento de la maduración de la cosecha, además de atenuar los efectos del estrés ambiental (Núñez, 1999; González- Olmedo et al., 2005b).

Los brasinosteroides como reguladores del crecimiento en vegetales son capaces de influir en diferentes procesos fisiológicos a muy bajas concentraciones, por lo que ha sido de gran interés científico-técnico el empleo de análogos de brasinoesteroides en la Biotecnología vegetal cubana, por sus efectos sobre la elongación, la división celular, el desarrollo vascular y el reproductivo (Núñez y Robaina, 2000).

El presente trabajo se desarrolló con el objetivo de evaluar diferentes concentraciones de brasinoesteroide MH5 en la aclimatización de brotes de Vriesea 
propagados in vitro, en Sistemas de Inmersión Temporal.

\section{Materiales y Métodos}

\section{Material vegetal}

$\mathrm{P}$ lantas cultivadas in vitro de un híbrido de Vriesea, Bromelia Ornamental, proveniente del Laboratorio Comercial SBW de Holanda. Se utilizaron grupos de 2-3 brotes, las hojas de los brotes se removieron y se cortaron a una altura de $1.5 \mathrm{~cm}$ desde la base.

El medio de cultivo fue el MS modificado. Para todos los experimentos, el $\mathrm{pH}$ del medio de cultivo se ajustó a 5.8 previo la esterilización por autoclave, la temperatura de esterilización fue $121{ }^{\circ} \mathrm{C}$ y una presión de $118 \mathrm{kPa}$. El tiempo de esterilización estuvo en correspondencia con el volumen de medio que se empleó en cada caso.

Se emplearon los Sistemas de Inmersión Temporal (SIT) previamente descritos por Escalona et al., (1999). Las condiciones del cultivo en el estante de inmersión temporal fueron de $25 \pm 1{ }^{\circ} \mathrm{C}$ de temperatura, un flujo de Fotones Fotosintéticos de $30-40 \mu \mathrm{mol} \cdot \mathrm{m}^{-2} . \mathrm{s}^{-1}$ y un foto período de 16 horas luz y 8 horas oscuridad.

Como indicadores de calidad se evaluaron la coloración de los brotes, altura de los brotes, número de hojas por brote, número de raíces por brote, así como la masa fresca y la masa seca. Para la determinación de la masa seca los brotes se colocaron durante 72 horas a 70 ${ }^{\circ} \mathrm{C}$ en estufa de convección (HSA) hasta llegar a un peso constante.

Efecto de la aplicación del análogo del Brasinoesteroides (MH5) en la supervivencia y en los indicadores morfológicos de calidad de las plantas, durante la aclimatización de los brotes de Vriesea

Para desarrollar este experimento se emplearon brotes de Vriesea proliferados y desarrollados según las condiciones experimentales establecidas anteriormente. Se emplearon SIT de $1500 \mathrm{ml}$ de capacidad y el volumen de medio de cultivo por explante fue de $42.8 \mathrm{ml}$. Al finalizar la fase de crecimiento en el medio MS todos los brotes se agruparon y se clasificaron en competentes (brotes mayores de $3 \mathrm{~cm}$ ) y no competentes (menores de $3 \mathrm{~cm}$ ), solo pasaron a la fase de aclimatización los brotes mayores de $3 \mathrm{~cm}$ de longitud.

Como sustrato se empleó una mezcla de zeolita + fibra de coco en una proporción $(1: 1, \mathrm{v}: \mathrm{v})$ en bandejas de 144 huecos (52.5 (L) x 29.5 (A) x 4 (A) cm) y se colocaron en una cámara de crecimiento. Con el empleo de un sistema automático de riego se logró una humedad relativa promedio de $85 \%$. Todas las mediciones ambientales se realizaron con un equipo CIRAS-2 (Sistema Portátil de Fotosíntesis, Europa, PP Systems, UK) acoplado a una cubeta universal (PLC6). La fase de aclimatización se desarrolló durante 45 días.

A todos los brotes se le aplicó polvo enraizador como inductor de la formación de raíces ex vitro. El polvo contenía ácido indol butírico (AIB) a una concentración de 492,12 $\mu \mathrm{mol} . \mathrm{mol}^{-1}$

En este experimento se evaluaron diferentes concentraciones de MH5: 0.0, 0.021, 0.107 y 0.216 $\mu \mathrm{mol}$. litroL $^{-1}$. El MH5 se asperjó foliarmente a los 7, 14,28 y 45 días de crecimiento en la fase ex vitro.

El análogo de brasinoesteroides MH5 es una formulación producida por el Centro de Estudios de Productos Naturales de la Facultad de Química de la Universidad de la Habana, la cual tiene como ingrediente activo un análogo espirostánico trihidroxilado de brasinoesteroides $\left(\mathrm{C}_{27} \mathrm{O}_{6} \mathrm{H}_{42}, \mathrm{MM}=462.606\right)$ en una concentración de $1 \mathrm{mg} \mathrm{ml}^{-1}$.

El experimento contó con cuatro tratamientos cada uno con tres repeticiones. En cada repetición se utilizaron 30 plantas para un total de 90 plantas por tratamiento. La supervivencia de las plantas se evaluó de forma dinámica a los 7, 14, 28 y 49 días. A los 49 días se evaluaron las siguientes variables: altura de la planta, número de hojas, número de raíces, masa fresca y masa seca.

Se utilizó el utilitario estadístico SPSS (versión 11.5 para Windows). Para el procesamiento estadístico de los datos se emplearon pruebas paramétricas y no paramétricas.

\section{Resultados y Discusión}

Efecto de la aplicación del análogo de Brasinoesteroides (MH5) en la supervivencia y en los indicadores morfológicos de calidad de las plantas en la aclimatización de los brotes de Vriesea

$E^{n}$ el Cuadro 1 se muestran los porcentajes de supervivencia de las plantas de Vriesea durante los 49 días de crecimiento en la fase de aclimatización. A partir de los catorce días comienza a declinar este indicador y los niveles más bajos de supervivencia se presentaron al final de la evaluación. A los 28 y 49 días se encontraron diferencias significativas entre la supervivencia de las plantas que se les aplicó el MH5 y el control sin aplicación de este regulador. Los porcentajes más bajos $(66 \%)$ lo presentaron las plantas que no recibieron la aplicación del análogo de brasinoesteroides al finalizar esta fase. 

Cuadro 1: Efectos de las aplicaciones del análogo de brasinoesteroides MH5 sobre la supervivencia de plantas de Vriesea durante los 49 días en la fase de aclimatización.

\begin{tabular}{|c|c|c|c|c|}
\hline \multirow{2}{*}{$\begin{array}{c}\text { Concentración de } \\
\text { MH5 } \\
\left(\mu \mathrm{mol.l}^{-1}\right) \\
\end{array}$} & \multicolumn{4}{|c|}{ Supervivencia (\%) } \\
\hline & 7 días & 14 días & 28 días & 49 días. \\
\hline 0 & 100 & 97 & $87.00 \mathrm{~b}$ & $66.00 \mathrm{~b}$ \\
\hline 0.021 & 100 & 98 & $96.00 \mathrm{a}$ & $88.00 \mathrm{a}$ \\
\hline 0.107 & 100 & 97 & $92.00 \mathrm{a}$ & $84.00 \mathrm{a}$ \\
\hline 0.216 & 100 & 99 & $95.00 \mathrm{a}$ & $87.00 \mathrm{a}$ \\
\hline ES & 0 & 7.32 & 7.84 & 8.16 \\
\hline Significación. & NS & NS & * & * \\
\hline
\end{tabular}

Medias con letras diferentes en una misma columna indican diferencias significativas para un grado de confiabilidad del $5 \%$ para la prueba de Student-Newman-Keuls. $(n=3)$

$\mathrm{Al}$ analizar los indicadores de calidad de las plantas a los 49 días de la aclimatización se observó un aumento significativo en el número de raíces y hojas de los brotes en los tratamientos donde se aplicó el MH5. La mayor concentración de brasinoesteroides provocó un aumento significativo de la masa fresca de los brotes en comparación con el valor alcanzado por este indicador en los grupos control y MH5 $0.021 \mu \mathrm{mol}^{1 .^{-1}}$. Con relación a la longitud de los brotes y la masa seca no se encontraron diferencias estadísticas entre los diferentes tratamientos (Cuadro 2).

\begin{tabular}{|c|c|c|c|c|c|}
\hline $\begin{array}{c}\text { Concentración de MH5 } \\
\qquad\left(\mu \mathrm{mol.}^{-1}\right)\end{array}$ & $\begin{array}{l}\text { Longitud/ } \\
\text { brote }\end{array}$ & $\begin{array}{l}\text { Número de } \\
\text { raíces/brote }\end{array}$ & $\begin{array}{l}\text { Número de } \\
\text { hojas/brote }\end{array}$ & $\begin{array}{c}\text { Masa fresca/ } \\
\text { brote (g) }\end{array}$ & $\begin{array}{c}\text { Masa seca/ } \\
\text { brote (g) }\end{array}$ \\
\hline 0 & 5.16 & $1.89 \mathrm{~b}$ & $11.3 \mathrm{~b}$ & $0.39 \mathrm{~b}$ & 0.03 \\
\hline 0.021 & 5.04 & $3.06 \mathrm{a}$ & $11.9 \mathrm{ab}$ & $0.34 \mathrm{~b}$ & 0.04 \\
\hline 0.107 & 5.05 & $2.62 \mathrm{a}$ & $12.1 \mathrm{ab}$ & $0.42 \mathrm{ab}$ & 0.04 \\
\hline 0.216 & 5.36 & $3.00 \mathrm{a}$ & $12.7 \mathrm{a}$ & $0.50 \mathrm{a}$ & 0.04 \\
\hline ES & 0.08 & 0.09 & 0.15 & 0.01 & 0.001 \\
\hline Significación & NS & $*$ & $*$ & $*$ & NS \\
\hline
\end{tabular}

Medias con letras diferentes en una misma columna indican diferencias significativas para un grado de confiabilidad del $5 \%$ para la prueba de Tukey $(n=30)$.

La transición desde el cultivo in vitro a la casa de cultivo es la fase final de cada esquema en la micropropagación. En el cultivo in vitro el ambiente se caracteriza por una alta humedad relativa, baja intensidad lumínica, limitado intercambio de gases, exposición a una fuente externa de carbono y por lo general altos niveles hormonales. Todos estos factores conducen a cambios estructurales y funcionales en la fisiología de las plantas. En las primeras semanas después de la transferencia al ambiente ex vitro, las plantas deben adaptarse a nuevas condiciones de crecimiento y tienen que desarrollar una fisiología normal y funcional del sistema radical (Debergh et al., 2000).

Para acelerar el proceso de aclimatización de las Vrieseas se utilizó el enraizamiento ex vitro con el empleo de polvo enraizador. Aunque en la micropropagación el enraizamiento in vitro es una práctica común, se ha podido comprobar que en general las raíces durante esta etapa son poco funcionales y tienen poca influencia en la aclimatización (Daquinta, 1998; Preece y Sutter, 1991).

Para evaluar el nivel de supervivencia de las 
plantas de Vriesea provenientes de la inmersión temporal se empleó como sustrato una mezcla de zeolita + fibra de coco con el objetivo de facilitar la aireación del entorno radical y favorecer la diferenciación de las raíces. La presencia de oxígeno en esta zona es un factor importante para la diferenciación y posterior emisión de las mismas. Por lo general las Bromeliaceae son muy sensibles a la falta de aireación en el sustrato (Baensch y Baensch, 1996).

A los 49 días de la aclimatización todos los tratamientos mostraron la presencia de raíces, lo cual apoya el efecto positivo que jugó la aplicación del polvo enraizador sobre esta variable morfológica.

Las plantas cuando van a la aclimatización ex vitro, presentan problemas de desecación debido a la pérdida de agua foliar y a la toma restringida de la misma por la incapacidad de las raíces en los primeros momentos. Esta es una de las principales causas de muerte de las plantas cuando se trasladan a condiciones ex vitro (Preece y Sutter, 1991).

En el proceso de aclimatización de las plántulas de Vriesea, las primeras muertes se detectaron a partir de los 28 días. Esta evidencia de adaptabilidad durante los primeros momentos de la salida ex vitro, presumiblemente se debe a la alta capacidad de almacenaje de agua y una mayor eficiencia en la utilización de la misma, característica fisiológica importante en la familia Bromeliaceae (Medina et al., 1989).

Por otra parte, las plantas que fueron asperjadas con MH5 mostraron la menor mortalidad. Esto pudiera estar relacionado con las propiedades anti-estrés de este regulador. Entre las diversas funciones que se le reconocen a los brasinoesteroides, están el aumento de la resistencia a las plagas y enfermedades y a diferentes factores de estrés como la alta salinidad, sequía, bajas y altas temperaturas y agentes químicos agresivos como plaguicidas y herbicidas (Sasse, 1997). La aplicación del análogo de brasinoesteroides MH5 a la concentración de $0.216 \mu \mathrm{mol} . \mathrm{L}^{-1}$ anuló el porcentaje de plantas de banano FHIA-18 con manchas en las hojas como consecuencia del estrés térmico a que estuvieron expuestas (González-Olmedo et al., 2005a). Tanto es esta especie como en piña se demostró el efecto antiestresante de MH5 por la reducción de los contenidos de prolina libre (González-Olmedo et al., 2005 b), probablemente en Vriesea indujo similares efectos que contribuyeron a los incrementos de la supervivencia de las plantas.

La aparición de plantas muertas al final de la etapa de aclimatización (49 días) pudiera estar relacionada con la inanición de las mismas por agotamiento de nutrientes en el sustrato $u$ otras condiciones necesarias a manipular en esta fase como son los niveles de luminosidad, temperatura, humedad relativa y fotoperíodo. Es evidente que la presencia de raíces a los 49 días debe garantizar la completa adaptabilidad de estas plantas a las condiciones de exteriores, por lo que la muerte por causa de estrés debe ser descartado. Es por ello que el proceso de aclimatización de estas plantas requiere de estudios posteriores con vistas a mejorarlo.

La aplicación de MH5 a las tres concentraciones que se ensayó, estimuló la formación de raíces y el número de hojas de las plantas de Vriesea, lo cual presupone un efecto sinérgico o aditivo con las auxinas en dicho proceso. En plántulas de banano FHIA18 provenientes del cultivo in vitro y expuestas a estrés térmico por altas y bajas temperaturas, la aplicación del MH5 a la concentración de $0.216 \mu \mathrm{mol}^{1 .^{-1}}$ aumentó significativamente el número de hojas, la altura, y la masa fresca y no tuvo ningún efecto en el número de raíces (González-Olmedo et al., 2005a).

Los brasinosteriodes promovieron el enraizamiento en tallos clonados de Matricaria chamomilla, en cortes de hipocotilo de soya, en posturas trasplantadas de Pinus radiata y en posturas o plantas de remolacha, trigo, maíz, tabaco y arroz. Además el tratamiento de cortes de Picea abies con (22S, 23S)-28-homobrasinólido aceleró la formación de raíces adventicias (Ronsch et al., 1993). Bishop y Yokota, (2001) informaron que los brasinoesteroides están involucrados en el gravitrofismo de la raíz de una forma dependiente con el ácido indol acético.

Los brasinoesteroides están involucrados en los procesos de alargamiento celular a través de sus efectos sobre la expresión de genes y/o la actividad de enzimas (Khripach et al., 2000). Estos compuestos actúan de manera sinérgica con las auxinas y aditivamente con giberelinas (Katsumi, 1985). También se acepta sinergismo o efectos aditivos entre los brasinoesteroides exógenos y otras hormonas de las plantas tales como las auxinas, giberelinas, citoquininas, $\mathrm{ABA}$ y etileno, principalmente en experimentos de elongación (Piqueras y Debergh, 1999).

Los cambios que se inducen en el crecimiento y desarrollo de las plantas por la aplicación de los brasinoesteriodes son el resultado de una cascada de eventos bioquímicos, los cuales pueden ser iniciados directamente sobre el genoma o a través de rutas que no impliquen la acción directa de los genes. Ambas vías asumen la participación de un sistema de mensajeros secundarios: una importante característica es la capacidad que tienen estos compuestos de actuar a extremadamente bajas concentraciones (Khripach et al., 2000). También en este experimento la dosis más baja de las ensayadas estimuló buenos resultados, en especial en la supervivencia y el follaje.

Por los resultados de este experimento es importante destacar que en el proceso de aclimatización de las plantas de Vriesea provenientes del cultivo en 
inmersión temporal, la aplicación de auxinas (polvo enraizador) y del brasinosteriode MH5 promovió la calidad morfológica de las mismas, lo cual se tradujo en un mayor número de raíces, número de hojas y masa fresca de los brotes. Se ha informado que las especies del género Vriesea en su hábitat natural son de muy lento crecimiento y desarrollo de allí que se requieran períodos de evaluación más prolongados. Los indicadores de calidad antes mencionados sin duda deben garantizar un adecuado proceso de crecimiento y desarrollo de las plantas cuando las condiciones propias de aclimatización sean establecidas.

\section{Conclusión}

$\mathrm{E}^{\mathrm{n}}$ el proceso de aclimatización de las plantas de Vriesea provenientes de SIT, la aplicación de auxinas (polvo enraizador) y del brasinoesteriode MH5 promovió la calidad morfológica de las mismas, lo cual se tradujo en un mayor número de raíces, número de hojas y masa fresca de los brotes.

\section{Literatura Citada}

Alves, M.; Guerra, P. 2001. Micropropagation for mass propagation and conservation of Vriesea firburgensis var. paludosa from microbuds. Journal of the Bromeliad Society 51(5): 202-212.

Baensch, U.; Baensch, U. 1996. Bromeliáceas en Flor. Tropic Beauty Publishers pp 236-245.

Bishop, G. J.; Yokota, T. 2001. Plant Cell Physiol. 42(2): 114-121.

Carneiro, L. A.; Arujo, R. F; Brito, G. J. M.; Fonseca, M. H. P. B.; Costa, A.; Crocomo, O. J. Y. ; Mansur, E. 1999. In vitro regeneration from leaf of Neorgelia cruenta ( R. Graham) L. B. Smit, an endemic bromeliad from Eastern Brazil. Plant Cell, Tissue and Organ Culture 55: 79-83.

Creelman, R. A; Mollet, J. E. 1997. Biosynthesis and action of jasmonate in plants. Annu. Rev. Plant Mol. Biol. 48: 355-381.

Daquinta, M. 1998. Propagación in vitro de la piña Ananas comosus (L) Merr. Tesis presentada en opción al grado científico de Doctor en Ciencias Agrícolas. 99 p.

Debergh, P. C.; Topoonyanont.; Van Huylenbroeck, J.; Moreira da Silva H. ; Oyaert, E. 2000. Preparation of microplants for ex vitro establishment. Proceeding of the internacional Symposium on methods and markers for quality assurance in micropropagation. Acta Horticulturae 530.

Escalona, M.; Lorenzo, J. C.; González, B.; Daquinta,
M.; González J L, Dejardins, Y, Borroto, C. G. 1999. Pineapple Ananas comosus (L). Merr micropropagation in temporary immersion systems. Plant Cell Reports 18(9): 723-748.

Gonzalez-Olmedo, J. L.; Fundora, Z., Molina, L. A., Abdulnour, J.; Desjardanis, Y.; Escalona, M. 2005a. New contributions to propagation of pineapple Ananas comosus (L) Merr in temporary immersion bioreactors. In vitro Cell, Dev. Biol.Plant.

González-Olmedo, J. L.; Córdoba, A. ; Aragón, C. E.; Pina. D.; Rivas, M.; Rodríguez, R 2005b. Efecto de un análogo de brasinoesteroides sobre plántulas de FHIA-18 expuestas a un estrés térmico. Infomusa 14(1): 18-23.

Katsumi, M. 1985. Interaction of a brassinosteroid with IAA and GA3 in the elongation of cucumber hypocotyls sections. Plant Cell Physiology 26: 615-625.

Khripach, V.; Zhabins, K. Z. ; Groot, A. 2000. Botanical Briefing. Annals of Botany. 86: 441-447.

Medina, E.; Olivares, E.; Díaz, M.; Van der Merwe, N. 1989. Metabolismo acido de crasuláceas en bosques húmedos tropicales. Monographs in Systematic Botany (Missouri Botanical Garden) 27: 56-67.

Mercier, H; Kerbauy, G. B. 1997. Micropragation of Ornamental Bromeliads (Bromeliacea). In: Y.P.S. Bajaj (ed) . Biotechnology in Agricultura and Foresty, vol. 40: High-Tech. and Micropropagation VI. Spriger Verlag. Pp 43-57.

Nuñez, M. 1999. Aplicaciones practicas de los brasinoesteroides y sus análogos en la Agricultura. Cultivos Tropicales 20 (3): 63-72.

Nuñez, M.; Robaina C M. 2000. Brasinoesteroides. Nuevos reguladores del crecimiento vegetal con amplias perspectivas en la agricultura. IAC. Campinas. Pp.83.

Pierik, R. L. M; Sprenkels, P. A. 1991. Micropropagation of Tillandsia cyanea. Journal of the Bromeliad Society 41: 9-12.

Piqueras, A.; Debergh, P. 1999. Morphogenesis in micropropagation chapter 15. Morphogenesis in plant tissue culture. Kluwer Academic Publisher. pp 443-462.

Preece, J. E; Sutter, E. G. 1991. Acclimatizacion in micropropagate plants to the greenhouse and field. En: Debergh, P. C. Zimmerman (eds). Micropropagation Technology ang Applications. Kluwer Academic Publisher. pp 71-93.

Ronsch, H. 1993. Influence of 22S, homobrassinoeide on rooting capacity and survival of adult Norway Spruce cuttings. Tree Physiology 12: 71-80.

Sasse, J. M. 1997. Recent progress in brassinosteroids research. Physiol. Plant. 100: 696-701. 